\title{
LOS PREJUICIOS Y LA IDENTIDAD PARA EL APRENDIZAJE DE LA LENGUA AIMARA DE LOS ESCOLARES DE HUAYTIRI, JAPOPUNCO Y TURUNTURU
}

\author{
PREJUDICE AND IDENTITY FOR AIMARA LANGUAGE LEARNING BY \\ SCHOOL STUDENTS FROM HUAYTIRI, JAPOPUNCO AND TURUNTURU
}

Ernesto Pino Nina', Saúl Domínguez Agüero ${ }^{2}$, Rubén Mamani Flores ${ }^{3}$, Gilberth Alán Benito Santos ${ }^{4}$

\section{RESUMEN}

Esta investigación trata sobre los prejuicios y la identidad para el aprendizaje de la lengua aimara de los escolares de las instituciones educativas de Huaytiri, Japopunco y Turunturu, en la provincia de Candarave, Región Tacna. Tiene como objetivo determinar cómo se relacionan los prejuicios y la identidad para el aprendizaje de la lengua aimara en los escolares de las comunidades mencionadas. La metodología empleada para la recolección de datos fueron dos encuestas. Se contó con una población de 62 estudiantes; y la muestra, 33 escolares de ambos géneros. Mediante la prueba de T de Student se comprobó que existe una relación directa entre los prejuicios y la identidad para el aprendizaje de la lengua aimara en los escolares de las tres comunidades andinas mencionadas.

Palabras clave: identidad social, aprendizaje, lenguaje.

\section{ABSTRACT}

This investigation had to do with prejudices and the identity for Aimara language learning by school students from Huaytiri's, Japopunco's and Turunturu's educational institutions, in the provinces of Candarave, in Tacna Region. The aim was determine how prejudices and identity for the learning of Aimara language by school students in the mentioned commumities are related. Two opinion polls were the methodology used for the collection of data. There was a population of 62 students and the sample was 33 students of both genders male and female. It was checked by means of Student's T test that exists a direct relation between prejudices and identity for Aimara language learning by the students in the three Andean mentioned communities.

Keywords: social identity, learning, language.

\section{INTRODUCCIÓN}

La raíz cultural de la región Tacna es el aimara; sin embargo, por la educación occidentalizada que se imparte, quienes definen la política de la educación nacional conciben que el Perú es un país monocultural; es decir, solo se privilegia la lengua española y la cultura occidental; no se brinda el tratamiento adecuado al aprendizaje de la lengua aimara ni otras lenguas originarias.

Desde la colonia persiste ese afán de castellanizar a los aimarahablantes, y como consecuencia el aimara se encuentra en peligro de extinción. Para que una lengua pueda presentar vitalidad, es importante el fortalecimiento de la identidad lingüística, desterrar los prejuicios que tanto daño causan.

Por ello en esta investigación se trabajó con niños y adolescentes de zona aimara, quienes estudian en instituciones educativas con escaso número de alumnos, ya que la mayoría tuvo que migrar a las ciudades. Se averiguó el nivel de prejuicios y el nivel de identidad lingüística en aras de impulsar la planificación lingüística y fomento de la educación

\footnotetext{
Magister en Ciencias con mención en Tecnología Educativa, Licenciado en Educación Lengua y Literatura. Facultad de Educación, Comunicación y Humanidades. Universidad Nacional.Jorge Basadre Grohmann.Correo electrónico:erpinonina33@hotmail.com

Magister en Literaturas Hispánicas. Licenciado en Literatura. Facultad de Educación, Comunicación y Humanidades. Universidad Nacional Jorge BasadreGrohmann.Correoelectrónico:sauldominguezzz@hotmail.com

'Licenciado en Ciencias de la Comunicación. Facultad de Educación, Comunicación y Humanidades. Universidad Nacional Jorge Basadre Grohmann

'Licenciado en Educación Lengua, Literatura y Gestión Educativo. Facultad de Educación, Comunicación y Humanidades. Universidad Nacional Jorge Basadre Grohmann
} 
intercultural.

Las normas o leyes para la recuperación de las lenguas originarias son favorables en el Perú; sin embargo, se persiste en el grave error de privilegiar la lengua española o el inglés, por eso es importante que las lenguas originarias del país sean tratadas con equidad respecto a otras lenguas de "prestigio". A pesar de tal situación, hay condiciones para superar los prejuicios y la falta de identidad con lo originario, en especial para fortalecer la vigencia del aimara.

Un estudio realizado en el año 2011 en la Facultad de Educación, Comunicación y Humanidades, Universidad Nacional Jorge Basadre Grohmann - Tacna, permitió determinar que más de la mitad de los estudiantes universitarios $(58,2 \%)$ se ubicó en el nivel poco prejuicioso. Por otra parte, "la mayoría de los estudiantes universitarios estuvo muy de acuerdo $(43,2 \%)$ y de acuerdo $(39,0 \%)$ con que no hay que dejar morir el aimara (...); asimismo, la mayoría de ellos se mostraron favorable y muy favorablemente en respetar y apoyar a esta lengua (...). Además, más del $67,0 \%$ se mostró favorable y muy favorable en hacer el esfuerzo para poder aprender el aimara bien (...); cl 45,9\% refirió que se debe aprender el aimara en el hogar y en todos los niveles educativos; conjuntamente, una quinta parte estuvo más decidido para tal propósito (...). En suma, más de la mitad de los estudiantes adoptaron una actitud favorable hacia el aimara" (Pino, 2011: 196 - 197).

Estos resultados indican que hay cierta identidad lingüística con el aimara, lo cual podría favorecer en la recuperación de esta lengua. Los factores que entren a tallar probablemente sean la pertenencia cultural, el parentesco aimara de los universitarios, las cuestiones históricas, entre otros.

Hay que recordar que la lengua es el marcador simbólico de identidad sociocultural (Prado, 2004). Toda lengua cumple esta función, mediante la cual el hablante puede sentirse miembro de un grupo y los miembros de otros grupos pueden ser discriminados. Las lenguas originarias como el aimara o de la amazonia tienen ese valor y sirven de símbolo de identidad lingüística y cultural, aunque algunos estratos de hispanohablantes discriminen a los hablantes de dichas lenguas por tener "menor prestigio social". Pero también hay grupos sociales que demuestran su lealtad hacia las lenguas originarias del país y se identifican fuertemente con su lengua materna.

Según Tusón (2003), los prejuicios son juicios u opiniones sesgadas, son insuficientes o defectuosos que suplantan a la verdad. Proliferan contra todo lo que es diferente, como otra lengua, otra persona, otra cultura. En su sentido negativo, los prejuicios son destructivos.

Por el contrario, la identidad es el proceso por el cual una persona acepta por propia voluntad una identidad del yo a diferencia del no yo; "se construye en un proceso de autovaloración, de concepción y definición de sí mismo y de la determinación propia de la pertenencia a un grupo étnico" (Apaza, 2007: 324). La autovaloración es en sí la toma de conciencia de un yo individual y colectivo; implica el conocimiento de sí mismo, aceptación, amarse a sí mismo y una posición activa de sí mismo. Pero los fenómenos de contactos lingüístico-culturales permiten comprender que las identidades han sufrido construcciones, cambios y variaciones. De allí que no sea posible hallar identidades lingüísticas o culturales puras. Todo se va transformando, se va recreando porque la lengua y la cultura son dinámicas.

Para Chryssochoou (citado por Martínez y Mora, 2008), "la identidad está formada por tres componentes: un elemento cognitivo (referente al conocimiento de sí mismo), un elemento afectivo (referente a las demandas, emociones, deseos y sentimientos ante los demás y ante sí mismo) y un elemento conductual (referente a acciones y conductas propias ante las de los otros). Según el autor, estos componentes son, de hecho, acciones y no meros aspectos de la identidad o de la personalidad. La identidad constituye la experiencia de ser que es el resultado de la interacción entre las acciones o conductas, de los afectos y del conocimiento... La identidad es considerada como una forma de representación social que media en la relación entre el individuo y el mundo social. Así, la identidad constituye el principio de organización de las relaciones simbólicas entre el individuo y $\mathrm{el} \mathrm{mundo} \mathrm{social,} \mathrm{en} \mathrm{el}$ sentido de que constituye el eslabón entre las organizaciones psicológicas (identificaciones y autocategorizaciones) y las regulaciones sociales. Con su participación activa en el mundo social (conociendo, reconociendo y demarcando) los individuos construyen su identidad, puesto que (co)construyen, afirman, negocian o cambian un sistema de conocimiento sobre el mundo y sobre sí mismos".

El presente artículo es parte de una investigación que se realizó en el Consejo General de Investigación de la Universidad Nacional Jorge Basadre Grohmann, por ello la información que se presenta es sintética. El problema fue el siguiente: ¿Cómo se relacionan los prejuicios y la identidad de los escolares de las instituciones educativas de Huaytiri, Japopunco y Turunturu para el aprendizaje de la lengua aimara en el año 2013? Se consignó como hipótesis general lo siguiente: Los prejuicios y la identidad para el aprendizaje de la lengua aimara guardan una relación directa en los escolares de Huaytiri, Japopunco y Turunturu. 


\section{MATERIALES Y MÉTODOS}

La investigación es básica y se caracterizó por presentar un diseño mixto. Por su ubicación en el tiempo, es una investigación transversal; por la forma en que se realizó, un diseño no experimental.

La población objeto de estudio fue un total de 62 estudiantes de ambos sexos y de diferentes años de estudio, de las instituciones educativas de las localidades de Huaytiri, Japopunco y Turunturu, en el año académico del 2013 de la provincia de Candarave, región Tacna.

Se tomó una muestra intencional aleatoria de 33 estudiantes, dado que en dicha zona hay escaso alumnado, tanto de la educación primaria (quinto y sexto grado) como de la secundaria (primero al quinto grado).

Una de las variables de estudio fue los prejuicios, cuyas dimensiones fueron los juicios y las creencias; la segunda variable, la identidad, contó con las siguientes dimensiones: lengua, límite geográfico, lo económico, lo social, conciencia, voluntad, lealtad y sentimiento de pertenecer.

Para la recolección de información, se empleó el método de la encuesta, la cual fue una adaptación a la de Amorrortu \& al. (2009). Dado que la investigación es de tipo no aplicada, no se consignaron equipos e instrumentos para su ejecución, pero se podría citar algunos equipos auxiliares como la laptop e impresora concretamente.

Para el procesamiento de la información sc utilizó el software estadístico SPSS, versión 18. Para el análisis de la información se empleó la estadística descriptiva y la inferencial; en el primer caso, la descripción de cuadros de frecuencias porcentuales y figuras de barras dobles, así mismo en el cálculo del coeficiente, el Rho de Spearman; en el segundo caso, para la prueba de hipótesis, el Test de Chi Cuadrado y la $\mathrm{T}$ de Student.

\section{Objetivos}

Determinar cómo se relacionan los prejuicios y la identidad de los escolares de Huaytiri, Japopunco y Turunturu para el aprendizaje de la lengua aimara.

Precisar el nivel de los prejuicios y de identidad de los escolares de Huaytiri, Japopunco y Turunturu para el aprendizaje de la lengua aimara.

\section{RESULTADOS}

Luego de haber procesado la información obtenida, se observó que el $42,4 \%$ de los 33 encuestados es de sexo masculino y el $57,6 \%$ femenino. En cuanto a sus edades, el $21,2 \%$ están comprendidos entre 10 y 12 años; el $54,5 \%$, entre 13 y 15 años; el $21,2 \%$, entre 16 y
18 años y el $3 \%$ tiene más de 19 años.

La primera lengua que aprendieron estos escolares es el castellano; así lo señaló el 87,9\%. El 9,1\% dijo que el aimara; y el 3,0\% no pudo precisar qué lengua fue la que aprendió primero.

El $66,7 \%$ de ellos no conoce otra lengua diferente al castellano, el 9,1\% de escolares después del español aprendió también la lengua aimara; el $6,1 \%$, el inglés y aimara; el 6,1\%; aimara y español; el $6,1 \%$, aimara y quechua; el $3,0 \%$, solo el inglés.

Respecto del entorno familiar, el 93,90\% de los jóvenes, dijo que su mamá habla el aimara; el papá, $78,80 \%$; la abuela, otro $78,80 \%$; el abuelo, el $75,80 \%$; los tíos, $72,70 \%$; otros familiares, el $36,40 \%$; y ningún familiar habla la lengua aimara, el 3,0\%. Si la mayoría representativa de los familiares de los escolares son aimarahablantes, los adolescentes tendrían que ser también aimarahablantes, pero ocurre todo lo contrario.

\subsection{Variable prejuicios}

Para conocer la percepción de los escolares sobre la lengua aimara, se presentó a los encuestados 16 preguntas en un cuestionario. He aquí algunas de ellas. En lo referente a si "el aimara es una lengua inútil", el $39,4 \%$ de los estudiantes está muy de acuerdo con que lo es una lengua inútil; el 30,3\% no está de acuerdo ni en desacuerdo; el $27,3 \%$ está de acuerdo y, solo el $3 \%$ está en desacuerdo. Es decir, la mayoría de los estudiantes de Huaytiri, Japopunco y Turunturu tienen la idea de que el aimara es una lengua inútil.

El hecho de que haya muchas lenguas como el aimara en el Perú, ¿perjudican el desarrollo del país? Ante esta pregunta, el 45,5\% de los encuestados está muy de acuerdo en que es así; el 30,3\% está de acuerdo, el $12,1 \%$ está en desacuerdo y el $12,1 \%$ restante no está de acuerdo ni en desacuerdo; por ende, la mayoría tiene la idea de que la existencia de muchas lenguas como el aimara en el Perú, perjudica el desarrollo del país.

Ante estos resultados, el $48,5 \%$ de los estudiantes considera que lo ideal es que a cada país debe corresponderle solo una lengua. El $24,2 \%$ se mostró ni de acuerdo ni en desacuerdo; el 18,3\%, de acuerdo; y, solo el 9,1\% estuvo en desacuerdo. En consecuencia, la mayoría de los estudiantes tienen el prejuicio de que lo ideal es el monolingüismo.

Respecto a la idea de si hay pocos aimarahablantes es porque es una lengua difícil de aprender, el $39,4 \%$ de los estudiantes respondió ni de acuerdo ni en desacuerdo; el $27,3 \%$ dijo estar de acuerdo con ello; el $18,2 \%$, en desacuerdo; y el $15,2 \%$, ni de acuerdo ni en desacuerdo. Por lo tanto, la mayoría de los encuestados tiene la idea de que si hay pocos aimarahablantes es porque es una lengua difícil de aprender. 


\section{La Vida y la Fistaria}

Pino, E. et al. Los Prejuicios y la Identidad para el Aprendizaje de la Lengua Aimara de los Escolares de Huaytiri, Japopunco y Turunturu

Ante el siguiente enunciado: "como el aimara no tiene escritura, es inferior a otras lenguas", el $57,6 \%$ de los escolares estuvieron muy de acuerdo con ello, el 27,3 dijo estar de acuerdo; el 9,1\%, ni de acuerdo ni en desacuerdo; y solo el $6,1 \%$, en desacuerdo. En consecuencia, la mayoría tiene el prejuicio de que el aimara es inferior a otras lenguas porque no tiene escritura. Existe desconocimiento que desde 1985 todos los países de habla aimara cuentan con un alfabeto unificado para la escritura, pero parece que no se ha difundido lo suficiente, lo cual no quiere decir que el aimara no tenga escritura.

Para complementar lo anterior, el $42,4 \%$ de los estudiantes no está de acuerdo ni en desacuerdo con que la lengua aimara no tiene gramática, el 27,3\% está muy de acuerdo con ello; el $21,2 \%$, de acuerdo; y el $9,1 \%$ está en desacuerdo. La mayoría tiene la idea de que la lengua aimara no tiene gramática. En principio, no hay ninguna lengua del mundo que no tenga gramática; por lo tanto, los estudiantes de las zonas andinas de Tacna en su mayoría evidencian prejuicios sobre este aspecto lingüístico.

Ante la idea de que "no es valioso para el Perú recuperar el aimara", el $66,7 \%$ de los escolares está muy de acuerdo con tal afirmación, el 27,3\% está de acuerdo; solo el $3,6 \%$, está en desacuerdo y el otro $3,6 \%$, no está de acuerdo ni en desacuerdo. En síntesis, la mayoría tiene el prejuicio de que no es valioso para el Perú recuperar el aimara. En estos resultados se aprecia una intención de autoeliminación lingüísticocultural de parte de los escolares. La occidentalización les ha convencido de que lo suyo no vale; mientras que lo español sí es valioso. Se estaría ante un autoprejuicio.

Esta situación se confirma también cuando a los escolares se les preguntó "¿Lo ideal sería que en el Perú solo se hable una lengua: el español?" El 45,5\% respondió estar muy de acuerdo con ello, el 30,3\% está de acuerdo, es decir, el $75,8 \%$ se mostró favorable hacia el monolingüismo español. Por otro lado, el $18,2 \%$ no está de acuerdo ni en desacuerdo y, solo el $6,1 \%$ se mostró en desacuerdo.

El prejuicio con el aimara se evidencia tambićn en que es considerado como una carga para el Estado. El $45,5 \%$ de los estudiantes dice estar muy de acuerdo con esa afirmación; el 30,3\% está de acuerdo; el 21,2\%, en ni de acuerdo ni en desacuerdo; y solo el $3 \%$ se mostró en desacuerdo.

Pero no solo eso, la gran mayoría de los encuestados considera que el aimara es una lengua desagradable. Así lo considera el $66,7 \%$ de los escolares que dice estar muy de acuerdo con ello, el 30,3\% está de acuerdo; solo el $6,1 \%$, se mostró en desacuerdo y el otro $6,1 \%$, en ni de acuerdo ni en desacuerdo.

Ante la idea de que "el aimara sigue siendo una lengua de serranos pobres", los dos tercios de los estudiantes $(66,7 \%)$ están muy de acuerdo con esa afirmación; el $18,2 \%$, de acuerdo; el $12,1 \%$, ni de acuerdo ni en desacuerdo; y solo el $3 \%$, en desacuerdo. La mayoría de los escolares tiene la idea que el aimara sigue siendo una lengua de serranos pobres.

La afirmación de que "el hablar aimara me hace sentir un ser inferior" permite corroborar de alguna manera el nivel de prejuicio de los escolares. El $36,4 \%$ está muy de acuerdo en que le resulta así; el $30,3 \%$ no está de acuerdo ni en desacuerdo. Por otra parte, el 24,2\% está de acuerdo, y solo el 9,1\% está en desacuerdo con dicha afirmación. En conclusión, la mayoría de los estudiantes piensan que el hablar aimara le hace sentir un ser inferior.

Finalmente, el $66,7 \%$ de los escolares encuestados está muy de acuerdo en que "en general, cree que el aprendizaje del aimara no es importante para su vida", el $24,2 \%$, dice estar de acuerdo; el 9,1\%, ni de acuerdo ni en desacuerdo; y solo el $6,1 \%$, en desacuerdo. Como se podrá apreciar, la mayoría de los estudiantes cree que en general el aprendizaje del aimara no es importante para su vida.

Después de haber constatado varios ítems se estableció el nivel de prejuicios para el aprendizaje de la lengua aimara. El $69,7 \%$ tiene alto nivel de prejuicios y el $30,3 \%$ tienen bajo nivel de prejuicios.

La educación escolarizada de los docentes y la educación informal de los padres de familia respecto a los escolares de Huaytiri, Japopunco y Turunturu no supieron orientar adecuadamente hacia la justa valoración de la lengua aimara. El alto porcentaje del nivel de prejuicios de los estudiantes revelaría dicha falencia.

Tabla $N^{\circ} 01$. Nivel de prejuicios para el aprendizaje de la lengua aimara de los escolares de Huaytiri, Japopunco y Turunturu, 2013

\begin{tabular}{ccc}
\hline Nivel & \multicolumn{2}{c}{ Estudiantes } \\
& Frecuencia & Porcentaje \\
\hline Bajo & 10 & 30,3 \\
Alto & 23 & 69,7 \\
Liotal & 33 & 100,0
\end{tabular}

Fuente: Elaboración propia a partir de la encuesta

\subsection{Variable identidad}

Para precisar el nivel de identidad de los escolares de las instituciones educativas de Huaytiri, Japopunco y Turunturu, se claboró un cucstionario de 14 preguntas. Entre las más relevantes se pueden considerar las siguientes:

Referente a la idea de que "me gusta ser parte del territorio aimara", los estudiantes de las tres comunidades respondieron así: El 33,3\% es indiferente, cl $30,3 \%$ están muy de acuerdo y el $21,2 \%$, de acucrdo. 
Quienes están en desacuerdo o no les gusta ser parte del territorio aimara suman el $9,1 \%$; y quienes están muy en desacuerdo, el $6,1 \%$. Es decir, a la mayoría de los escolares les gusta ser parte del territorio aimara; aunque a un tercio del total les resulta dudoso aceptar el sentirse a gusto en dicho territorio.

Ante la afirmación, "para poder respetar los derechos de los aimarahablantes, los profesores deben hablar el aimara", el 30,3\% de los estudiantes encuestados consideran que así debería ser; el $27,3 \%$ son indiferentes. Su posición es no estar de acuerdo ni en desacuerdo. Mientras que el 18,2\% está muy de acuerdo con que sus docentes hablen el aimara. Quienes son contrarios a esta exigencia representan el $15,2 \%$ que expresan su desacuerdo; y el $9,1 \%$ dicen estar muy en desacuerdo. En otros términos, una mayoría relativa de los escolares $(48,5 \%)$ considera que sus profesores deben hablar el aimara para que sus derechos sean respetados.

Respecto al enunciado "yo estoy contento de ser aimara", la mayoría de los escolares encuestados en las tres instituciones educativas se sienten contentos de ser aimaras. Así lo señalan el 39,4\% que dicen estar muy de acuerdo y el $12,1 \%$ de acuerdo, haciendo un total de $51,5 \%$ del total. Quienes no están contentos ni descontentos con ser aimaras representan el 27,3\%. Los que están en desacuerdo suman el 18,2\%; y los muy en desacuerdo, el $3 \%$.

En cuanto a la pregunta ¿es justo pedir que se sepa la lengua aimara en todo Tacna?, la mayoría de los escolares respondió favorablemente. El 33,3\% está de acuerdo con la pregunta y el $21,2 \%$, muy de acuerdo, lo que hace que un total de $54,5 \%$ esté a favor de que en toda la Región se hable dicho idioma. Los indiferentes suman el $36,4 \%$, que no están de acuerdo ni en contra; y los que están muy en descuerdo suman el 9,1\%.

Referente a la idea de que no se debe dejar morir la cultura aimara, el 51,5\% afirmó estar muy de acuerdo con ello; el 30,3\% está de acuerdo; es decir, más del $80 \%$ es consciente o al menos siente que se debe preservar la cultura aimara. Los indiferentes suman el $15,2 \%$ y quienes consideran que debe morir la cultura aimara apenas significan el $3 \%$.

Si bien la mayoría de los escolares encuestados dice que se debe preservar la cultura aimara, ante la idea de que "para que la lengua aimara no muera, es necesario extender su uso, aunque pueda entenderse como una imposición", el 39,4\% de los encuestados se mostró indiferente ante dicha afirmación; el 30,3\%, de acuerdo y el $12,1 \%$, muy de acuerdo. Quienes están en desacuerdo suman el $12,1 \%$; y muy en desacuerdo, el $6,1 \%$. En sintesis, una mayoría relativa del $42 \%$ considera que se debe extender el uso de la lengua aimara para que esta no muera.

¿Los niños de la Región Tacna deben aprender el aimara? La respuesta ante esta pregunta es la siguiente: El 39,4\% está de acuerdo y el $27,3 \%$, muy de acuerdo. Los indiferentes son relativamente significativos. El $30,3 \%$ no tiene una posición clara y responde que no está de acuerdo ni en desacuerdo. Solo el $3 \%$ considera que los niños de la región no deben aprender el aimara.

$\mathrm{Y}$ ¿haría el esfuerzo de aprender a escribir bien en la lengua aimara? La mayoría de los escolares encuestados respondió positivamente. El 33.3\% dijo estar de acuerdo en realizar el esfuerzo y el $27,3 \%$ muy de acuerdo. En este caso, los indiferentes son significativos aunque no determinantes. $\mathrm{El} 21,2 \%$ dice estar ni de acuerdo ni en desacuerdo, es decir, no tiene mucho interés en aprender a escribir bien en la lengua aimara. Quienes están en contra están representados por el $12,1 \%$, que dice estar en desacuerdo; y el $6,1 \%$, muy en desacuerdo.

Respecto a la idea de que "mi voluntad es que la cultura aimara siga por siempre", el $45,5 \%$ de los escolares concuerda con dicha afirmación, y eso refleja de alguna manera su identidad con lo suyo. Además, el $15,2 \%$ se muestra muy de acuerdo con tal afirmación. El $24,2 \%$ asumió una posición ambivalente; el 12,1\% manifestó su desacuerdo con que la cultura aimara siga por siempre; y el $3 \%$ respondió estar muy en desacuerdo con dicha proposición.

Ante la pregunta de que "mis antepasados fueron aimaras; yo también pertenezco a la nación aimara", la mayoría de los escolares es consciente de que es así. El 30,3\% respondió estar de acuerdo con la. afirmación propuesta; y el $24,2 \%$ dijo estar muy de acuerdo. Los indiferentes suman casi un tercio, el $27,3 \%$, lo que es una constante y un reflejo de su desconcierto o falta de identidad y sentido de pertenencia a un grupo social o nación. Quienes perdieron totalmente la identificación con sus antepasados, representan el 12,1\%, que respondió estar en desacuerdo y desconocen o niegan que sus antepasados hayan sido aimaras y menos aceptan su pertenencia a la nación aimara. Y los que están muy en desacuerdo suman el $6,1 \%$ del total.

Respecto a la afirmación de que "debemos aprender $y / o$ practicar todas las manifestaciones culturales aimaras", el $33,3 \%$ de los encuestados asumió la postura de ni de acuerdo ni en desacuerdo. Su falta de definición refuerza su falta de identidad con lo suyo que también se ha reflejado en los ítems precedentes. En cambio, el $30,3 \%$ de los escolares estuvieron de acuerdo con que deben aprender y practicar las manifestaciones culturales aimaras. Más firme en esta posición lo constituye el $24,2 \%$ que respondió estar muy de acuerdo con dicha proposición. Quienes están en desacuerdo, es decir, no les interesa aprender y preservar las manifestaciones culturales de 


\section{La Vida y la Ftistoria}

Pino, E. et al. Los Prejuicios y la Identidad para el Aprendizaje de la Lengua Aimara de los Escolares de Huaytiri, Japopunco y Turunturu

su nación representan el $9,1 \%$ de los encuestados; y los que afirman estar muy en descuerdo suman apenas el $3 \%$.

En cuanto a la pregunta de que "en general, me identifico completamente con la lengua y cultura aimaras", el 33,3\% de los escolares se mostró ni de acuerdo ni en desacuerdo. Esto, sin duda, es un signo claro de su falta de identidad lingüístico-cultural. Por otro lado, el $24,2 \%$ sostuvo estar en desacuerdo con identificarse completamente con la lengua y cultura aimaras; quienes rechazaron con mayor énfasis tal posibilidad suman apenas el $3 \%$. En cambio, el 21,2\% afirmó estar de acuerdo con el enunciado; y los que están muy de acuerdo sumaron el 18,2\%. En síntesis, una mayoría relativa valora y se identifica completamente con su lengua y cultura.

Después de haber examinado varios ítems en torno a la segunda variable, se logró determinar que el nivel de identidad para el aprendizaje de la lengua aimara es bajo. Así se determinó que el $72.7 \%$ de los estudiantes de las instituciones educativas de Huaytiri, Japopunco y Turunturu se ubicaron en el nivel bajo; mientras que quienes evidencian un nivel alto representan el $27,3 \%$ del total de los encuestados.

Tabla $\mathrm{N}^{\circ} 02$. Nivel de identidad para el aprendizaje de la lengua aimara de los escolares de Huaytiri, Japopunco y Turunturu, 2013

\begin{tabular}{ccc}
\hline Nivel & \multicolumn{2}{c}{ Estudiantes } \\
& Frecuencia & Porcentaje \\
\hline Bajo & 24 & 72,7 \\
Alto & 9 & 27,3 \\
Total & 33 & 100,0 \\
\hline
\end{tabular}

Fuente: Elabomción propia a partir de in encuesta

En función de los dos cuadros anteriores que revelan el nivel de prejuicios y de identidad, se pudo comprobar la hipótesis general en los siguientes términos: Existe una relación directa, según la prueba de T de Student, entre los prejuicios y la identidad para el aprendizaje del aimara de los escolares de Huaytiri, Japopunco y Turunturo, para un nivel de confianza del $95 \%$.

\section{DISCUSIONES}

En la presente investigación se ha podido constatar que los escolares de las comunidades andinas de Huaytiri, Japopunco y Turunturu se ubican en el alto nivel de prejuicios hacia la enseñanza-aprendizaje de su propia lengua que es el aimara. Este resultado debe invitar a los que dirigen los destinos de la educación en la Región Tacna a que no se siga postergando el aprendizaje de esta lengua milenaria.
En una posibilidad extrema, el alto porcentaje de prejuicios podría desencadenar en la extinción de la lengua y cultura aimaras, que son los patrimonios de toda la humanidad.

En un estudio realizado a los estudiantes universitarios de lo que actualmente se denomina la Facultad de Educación, Comunicación y Humanidades de la Universidad Nacional Jorge Basadre Grohmann de Tacna (Pino, 2011: 113), se pudo determinar que respecto a los prejuicios lingüísticos en la Escuela de Ciencias de la Comunicación destacó el nivel poco prejuicioso $(61,7 \%)$; lo mismo ocurrió en la Escucla de Educación (56,6\%). Si se compara este resultado con el de las instituciones educativas de la zona andina de Tacna, son diametralmente opuestos: Los estudiantes universitarios se ubican en el bajo nivel de prejuicios; mientras que los escolares de las localidades de Huaytiri, Japopunco y Turunturu, en alto nivel.

Se pensaba que mientras más alejados estén las personas de las grandes ciudades; habría mayor probabilidad de preservar su propia lengua y cultura; pero no es así. Tal vez sea por la ilusión de que produce la tecnología de la cultura occidental, la lengua y cultura aimaras no es muy bien vista por la misma comunidad de usuarios. En otros términos, la cultura occidental ha sometido a la cultura nativa hasta el punto de que los de esta última rechacen lo propio, lo desacrediten.

Así como el euskera que tiene muy poca o nula presencia en el contexto de los castellanohablantes de España (Amorrortu et al., 2009), en el caso de los escolares de las comunidades altoandinas de Tacna que fucron objeto de estudio, se podría decir que la lengua aimara también tiene poca presencia en sus múltiples funciones debido al alto nivel de prejuicios.

Con respecto a la identidad, en el caso de los escolares de Huaytiri, Japopunco y Turunturu pareciera marchar autodestructivamente, salvo con algunas excepciones. Esta negación de la propia lengua y cultura podrían ser alentadas incluso por los propios padres de familia. El bajo nivel de identidad que evidencian los estudiantes de los tres colegios en cuanto a su propia lengua y cultura, es una muestra de la aculturación, la alienación o negación de sí. Lo correcto sería como ocurre en la cultura mapuche, según refiere Carihuentro (2007), que una persona que tiene buen dominio y uso del lenguaje es considerado sabio porque la lengua mapuche es la base del conocimiento propio, de la identidad y la formación en general.

El nacionalismo lingüístico del español (Moreno, 2010) que excluyó a las lenguas originarias como el aimara, ha conducido a la negación de los derechos elementales de las comunidades andinoamazónicas. Esta situación ha llevado a un autodesprecio de los escolares hacia su propia lengua y 
cultura. Las lenguas son como organismos vivos que nacen, se desarrollan y mueren. Sobre el caso aimara, según los datos que han aportado los escolares de estas tres instituciones educativas, se podría deducir que está en peligro de extinción. Y cuando se pierde una lengua o una palabra, como bien lo dice Rengifo (2001), se habrá perdido una manera peculiar de relación humana con la realidad.

\section{CONCLUSIONES}

La investigación ha permitido arribar a las siguientes conclusiones:

El $69,7 \%$ de estudiantes de las instituciones educativas de Huaytiri, Japopunco y Turunturu presentan un alto nivel de prejuicios para el aprendizaje de la lengua aimara; mientras que el restante, $30,3 \%$, presenta un bajo nivel de prejuicios. Además, se determinó que el $72,7 \%$ de los estudiantes tienen un bajo nivel de identidad y quienes evidenciaron un nivel alto de identidad representan el $27,3 \%$ restante.

Los prejuicios y la identidad para el aprendizaje de la lengua aimara guardan una relación directa en los escolares de Huaytiri, Japopunco y Turunturu, para un nivel de confianza del $95 \%$. A mayor nivel de prejuicios hacia el aimara, se evidenció menor nivel de identidad. Por lo tanto, si se procura fortalecer la identidad lingüístico-cultural, primero se deben revertir los prejuicios lingüísticos existentes.

Ante esta situación se sugiere que en las instituciones educativas de Huaytiri, Japopunco y Turunturo, se debe realizar campañas de promoción y dignificación del aimara con el fin de que esta lengua perdure para otras generaciones. Se debe hacer entender a la comunidad aimara que los prejuicios que expresan de su propia lengua y cultura podrían llevarles a un autoexterminio cultural. Se debe fortalecer la identidad aimara a nivel regional; $\mathrm{y}$ a nivel nacional, también forjar la identidad nacional.

Se debe promocionar con más énfasis la educación intercultural bilingüe en la población que fue objeto de investigación. En el currículo de estudios, las autoridades, los docentes, los alumnos y padres de familia deben contemplar prioritariamente la enseñanza-aprendizaje de la lengua aimara con el fin de revalorar su legado cultural. No hay que olvidar que la lengua aimara es portadora de la ciencia, la tecnología, el arte y la religiosidad andinas.

\section{REFERENCIAS BIBLIOGRÁFICAS}

AMORRORTU, E.. ORTEGA, A., IDIAZABAL, T. \& BARREÑA, A. (2009). Actitudes y prejuicios de los castellanohablantes hacia el euskera. Vitoria-Gasteiz, Gobierno Vasco, $354 \mathrm{pp}$.

APAZA, 1. (2007). Estudios de lingüistica $y$ sociolingüistica andina. La Paz: Instituto de Estudios Bolivianos.

BRIGGS, L. T. (1993). El idioma aymara. Variantes sociales y regionales. La Paz: Ediciones $\mathrm{LCA}$.

CALLO,S. (2007). Diccionario aymara castellano/ Kamisaraki. Tacna: Perúgráfika SAC.

CARIHUENTRO, S. (2007). Saberes mapuches que debiera incorporar la educación formal en contexto interétnico $e$ intercultural según sabios mapuche. Tesis para optar el grado de magister. Santiago - Chile: Universidad de Chilc, Facultad de Ciencias Sociales, Escuela de Postgrado, $172 \mathrm{pp}$.

GAMERO, Estela (2000). Wiñaya aymara aru/ Eterna voz aymara. Tacna: "El Tigre".

MARTINEZ, H. y MORA, E. (2008). La identidad lingüística y los trastornos del habla. [Online], Boletín de Lingüística, Vol. 20, № 29, Págs. 85-101, Caracas, jun. 2008. Disponible cn:

http://www.scielo.org.ve/scielo.php?script $=$ sci arttext\&pid $=$ $\underline{\text { S0798-97092008000100004\&lng }=\mathrm{es} \& \mathrm{nrm}=\mathrm{iso}}$

[Consultada el 12 abril del 2013].

MINTDU (2013). IIacia una educación intercultural bilingüe de calidad. Lima: Ministerio de Educación.

MORENO, J. C. (2002). La dignidad y la igualdad de lenguas. (Segunda edición). Madrid: Alianza Editorial.

PRADO, Josefina (2004). Didáclica de la lengua y la lileratura para educar en el siglo XXI. Madrid: Editorial La Muralla.

PINO, E. (2011). Los prejuicios y las actitudes de los estudiantes de la Facultad de Humanidades de la Universidad Nacional Jorge Basadre Grohmann de Tacna, para el aprendizaje de la lengua aymara en el año 2011. Tesis para optar el grado de magister. Tacna: Universidad Nacional Jorge Basadre Grohmann, Escuela de Post Grado, 246 pp.

TUSÓN, J. (2003). Los prejuicios lingüisticos. (Segunda cdición). Barcelona: Ediciones Octacdro. 